\title{
The gamma and neutron monitor counters for the MICADO project
}

\author{
Paolo Finocchiaro ${ }^{1}$, Luigi Cosentino ${ }^{1}$, Quentin Ducasse ${ }^{2}$, Sergio Lo $\mathrm{Meo}^{3}$, Fabio Longhitano ${ }^{1}$, Carmelo \\ Marchetta $^{1}$, Antonio Massara ${ }^{1}$, Alfio Pappalardo ${ }^{1, \#}$, Giuseppe Passaro ${ }^{1}$, Salvatore Russo ${ }^{1}$ \\ ${ }^{1}$ INFN Laboratori Nazionali del Sud, 95123 Catania, Italy \\ ${ }^{2}$ CEA, DES, IRESNE, Nuclear Measurement Laboratory, Cadarache, F- \\ 13108 Saint-Paul-lez-Durance, France \\ ${ }^{3}$ ENEA Centro Ricerche, 40129 Bologna, Italy \\ ${ }^{\#}$ Current address: Extreme Light Infrastructure Nuclear Physics, 077125 \\ Magurele, Romania
}

finocchiaro@lns.infn.it

\begin{abstract}
In the framework of the MICADO (Measurement and Instrumentation for Cleaning And Decommissioning Operations) European Union (EU) project, aimed at the full digitization of low and intermediate-level radioactive waste (radwaste) management, a set of 32 solid state thermal neutron detectors, named SiLiF, and 36 gamma-ray counters based on a scintillating fiber readout at each end by a silicon photomultiplier, named SciFi, have been built and characterized. MICADO project encompasses a complete active and passive characterization of the radwaste drums with neutrons and gamma rays, followed by a longer-term monitoring phase. The detectors described are suitable for the monitoring of nuclear materials and can be used around radioactive waste drums possibly containing small quantities of actinides, as well as around spent fuel casks in interim storage or during transportation. Suitable polyethylene moderators, for the SiLiF detectors, can be exploited to better shape the detector response to the expected neutron spectrum, according to Monte Carlo simulations that were performed. The SciFi detectors were thoroughly tested with a ${ }^{22} \mathrm{Na}$ and a ${ }^{137} \mathrm{Cs}$ gamma-ray sources. Both detectors described were tested with an intense AmBe source of neutron and gamma ray. The results are satisfactory and show a quite uniform and reproducible behavior. The next step will be the test in a real environment.
\end{abstract}

Keywords - gamma-ray counters; neutron counters; radwaste management; radwaste monitoring.

\section{INTRODUCTION}

The MICADO (Measurement and Instrumentation for Cleaning and Decommissioning Operations) Euratom (EU) project is aimed at the full digitization of low-level and intermediate-level radioactive waste (radwaste) management [1,2]. Following a complete active and passive characterization of the radwaste drums with neutrons and gamma-rays, the project contemplates a longer-term monitoring phase in the Work Package 7 by means of low-cost dedicated detectors for neutrons, named SiLiF [3], and for gamma-rays, named SciFi [4]. The proposed system for the online real-time monitoring consists of an array of many radiation sensors to be deployed all around a number of radioactive waste drums, in order to collect counting-rate data in real time and to make them available to a software platform named DigiWaste. A continuous automatic monitoring of the radwaste drums after their characterization represents an added value in terms of safety and security, and the availability of continuous streams of counting-rate data around each drum would be a comfortable tool toward the transparency, which now more than ever is a relevant topic of the nuclear industry [1,3-8]. As an evolution of the proof-ofprinciple systems discussed in [5-8], the detectors described here will be part of a prototype system which will be installed in a few real radwaste storage sites to prove its effectiveness. To be suitable for mass deployment these detectors must be small, reasonably inexpensive, robust, easy-to-use and reliable. Furthermore, the sensors must be configurable in a modular and scalable fashion, so that one can tailor the system to small, medium, and large-scale storage configurations. The proposed monitoring system, indeed, is based on detectors which can be easily installed and/or reassembled in different geometrical configurations, as they are mechanically very simple and are based on commercial electronics.

The radiological monitoring of radwaste must be based on the measurement of gamma-rays and neutrons because these are penetrating and thus more easily detectable out of the drums.

The SciFi gamma and SiLiF neutron monitor counters built and tested are described. The SciFi sensors were thoroughly tested with a ${ }^{22} \mathrm{Na}$ and a ${ }^{137} \mathrm{Cs}$ gamma-ray sources. Both detectors were tested with an intense AmBe source of neutron, which also emits a wide spectrum of gamma-rays thus somehow resembling a real radwaste drum. The results of the tests are shown. 


\section{SiLiF NEUTRON DETECTOR}

The operating principle of the SiLiF neutron detector, as a viable alternative to ${ }^{3} \mathrm{He}$ tubes [9], is straightforward: following a thermal neutron capture by ${ }^{6} \mathrm{Li}$, the ${ }^{7} \mathrm{Li}$ compound nucleus decays into an alpha particle $\left({ }^{4} \mathrm{He}\right)$ and a triton $\left({ }^{3} \mathrm{H}\right)$, emitted back-to-back with high energy according to the reaction

$$
\mathrm{n}+{ }^{6} \mathrm{Li} \rightarrow{ }^{3} \mathrm{H}(2.73 \mathrm{MeV})+{ }^{4} \mathrm{He}(2.05 \mathrm{MeV})
$$

Semiconductor detectors, e.g., silicon diodes, can be used in combination with a neutron reactive film, usually made of ${ }^{6} \mathrm{Li}$ or ${ }^{10} \mathrm{~B}$ and called neutron converter. Such a film converts thermal neutrons into charged particles which are then detected by the silicon diode. The cross section at thermal neutron energy decreases with the inverse of the neutron velocity. In such a scheme fast neutron can also be detected by surrounding the detector with a suitable moderator box, typically made from polyethylene, which slows neutrons down to thermal energy. The ${ }^{6} \mathrm{Li}$, following neutron's capture, has a unique decay channel with no gamma rays emitted and with high kinetic energy, and decays into easy-to-detect particles [10]. Since ${ }^{6} \mathrm{Li}$ is chemically very reactive it was decided to employ ${ }^{6} \mathrm{LiF}$, a stable salt enriched at $95 \%$ in ${ }^{6} \mathrm{Li}$, that can be deposited by evaporation onto a suitable substrate. Instead of the direct deposition onto the silicon diode it was chosen to use independent detector and converter, which allows a better modularity and reconfigurability [11].

In Fig. 1 it is possible to see schematically the detection of neutrons by a $\mathrm{SiLiF}$ detector.

The detector configuration, shown in Fig. 2, consists of the double-sided silicon diode MSX09-300 sandwiched between two ${ }^{6} \mathrm{LiF}$ converter layers deposited onto carbon fiber substrates.

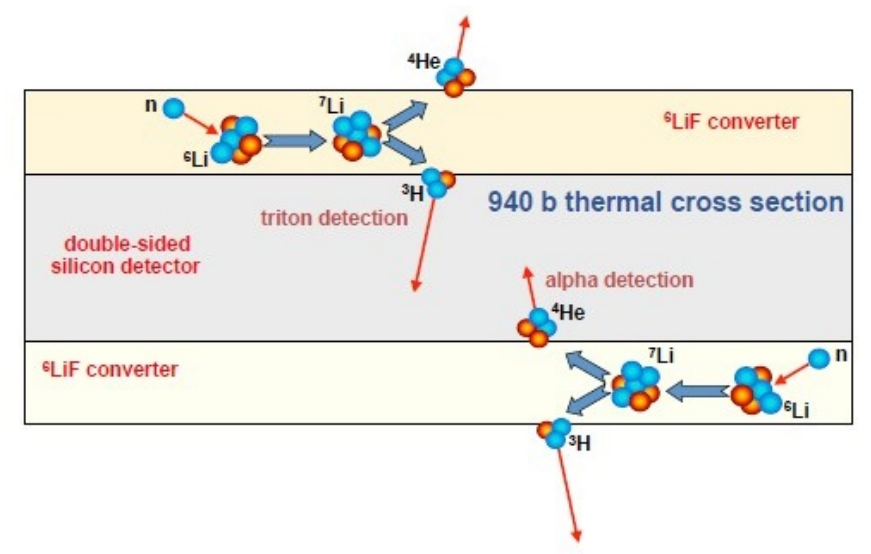

Fig. 1. The SiLiF scheme neutron detection.

To increase the detection efficiency for faster neutrons one makes use of a moderator. For the purposes of the MICADO project, the focus was on optimizing the moderator for neutrons with energy ranging from thermal to a few $\mathrm{MeV}$. The moderator of $4 \mathrm{~cm}$ thickness, shown in Fig. 3a, gave the best results and was replicated in 32 units. The detector in its standard configuration, with $10 \times 10 \times 10 \mathrm{~cm}^{3}$ size, is shown in Fig. 3c.

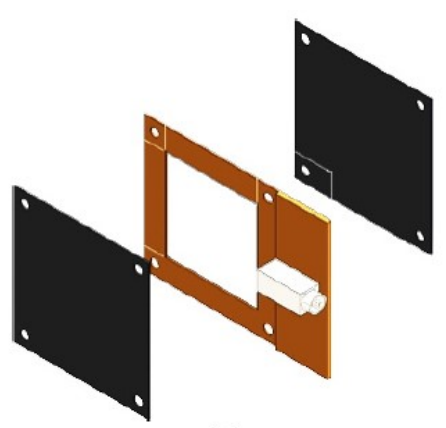

(a)

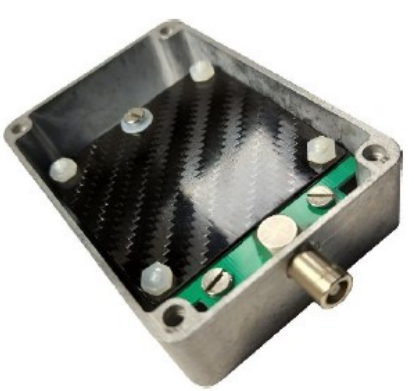

(b)
Fig. 2. (a) The SiLiF detector arrangement; (b) a real detector assembled inside its aluminium box.

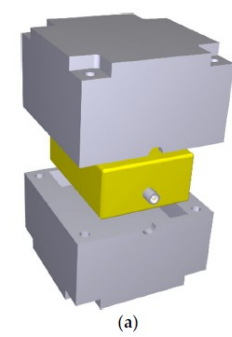

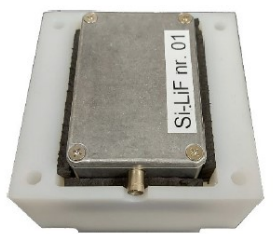

(b)

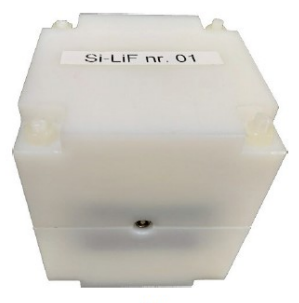

(c)
Fig. 3. (a) 3D sketch of the SiLiF detector; (b) a SiLiF hosted in a half moderator; (c) final assembling of a SiLiF.

\section{A. Test results}

To test and characterize the SiLiF detectors, an intense AmBe source $\left(2.2 \times 10^{6}\right.$ neutrons/s) was used, installed in an experimental hall at the INFN Laboratori Nazionali del Sud (LNS). The source is surrounded by a first polyethylene case followed by $30 \mathrm{~cm}$ thick paraffin that slow down the high energy neutrons it emits.

The first set of measurements that was performed was a preliminary check that the detectors were operational and behaved as expected. Each detector in turn, inside its moderator, was placed on a small cart and positioned in front of the source just out of the box with the open door (Fig. 4a).
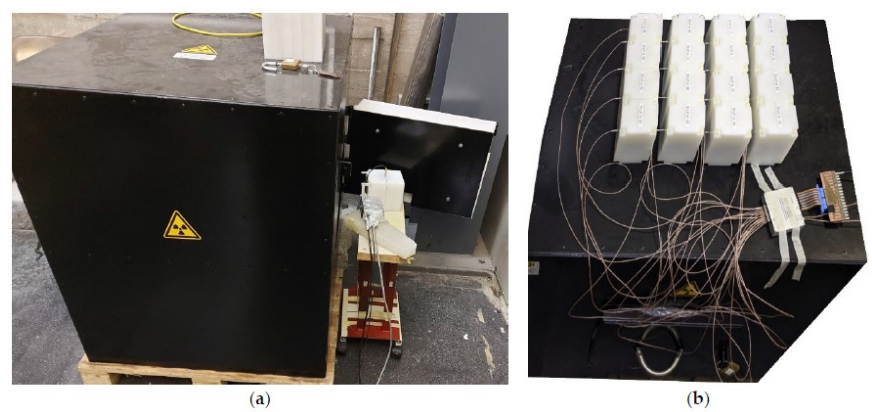

Fig. 4. (a) The AmBe neutron source box with a SiLiF detector in the front position. (b) The $4 \times 4$ array of $\mathrm{SiLiF}$ detectors in the top position.

A final test was performed using simultaneously 16 detectors aimed at measuring a low intensity flux of fast neutrons. To this purpose the detectors were arranged in a square $4 \times 4$ array in the top position upon the neutron source box (Fig. 4b). The detector was biased at $50 \mathrm{~V}$, in order to operate in full depletion regime and be sensitive on both faces. 
A charge preamplifier, with a nominal gain of $25 \mathrm{mV} / \mathrm{MeV}$, connected to a spectroscopy amplifier whose output was sent to an Amptek MCA8000A multichannel analyzer was used.

The superposition of the triton and alpha deposited energy, plus the effect of the emission from different depths/angles, produces a characteristic spectrum shape, as can be observed in Fig. 5, that also proves that all the detectors were working properly. The measurements were compared with Fluka [12] simulations giving an excellent agreement.

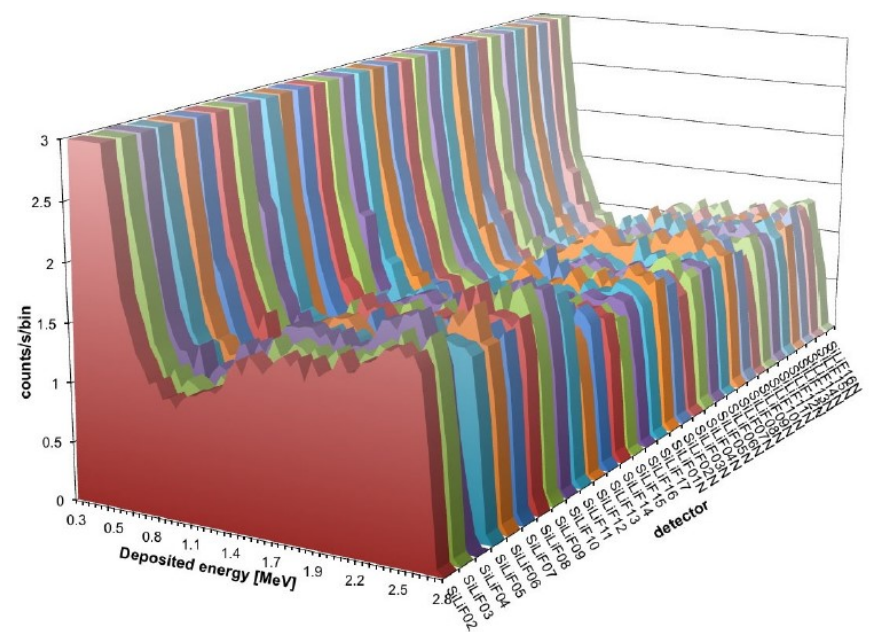

Fig. 5. The characteristic spectrum shape, measured for all the SiLiF detectors in the front position

In Fig. 6 the result of the final test where have been compared the (a) GEANT4 simulated and (b) evaluated fluxes, and still the (c) GEANT4 simulated and (d) measured counting rates. Unfortunately, due to a broken channel in the electronics, a centrally located detector did not provide useful data (white square with 0.00). It is however possible to state that the measurements have a good agreement with the simulations.
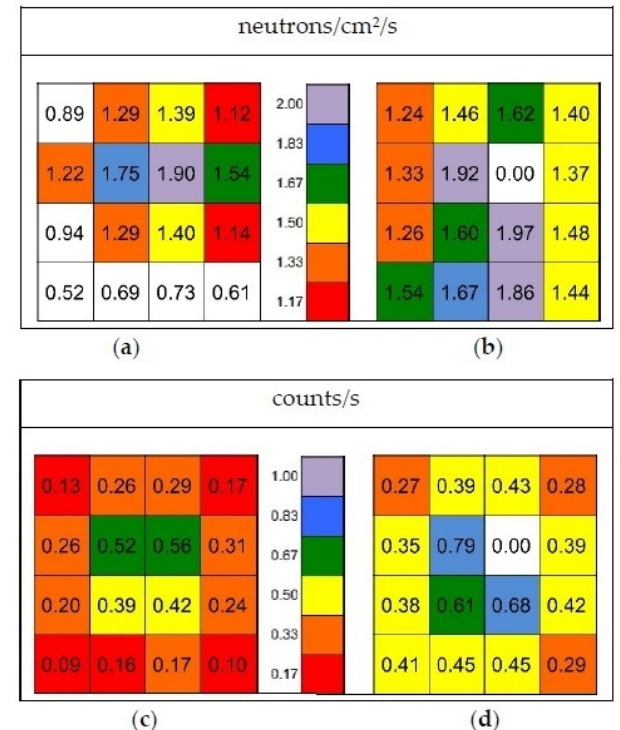

Fig. 6. (a) Total neutron flux simulated in the top position in correspondence of the $4 \times 4 \mathrm{SiLiF}$ array; (b) The measured total neutron flux with the $4 \times 4$ array. (c) The simulated neutron counting rates. (d) The measured neutron counting rates.

\section{SCIFI GAMMA-RAY DETECTOR}

To monitor the gamma radiation coming from the radioactive waste drums (height 76 or $88 \mathrm{~cm}$ ), we opted for a simple and robust design of the detectors. A $3 \mathrm{~mm}$ diameter scintillating fiber was allocated inside a $2 \mathrm{~cm}$ diameter and 1 $\mathrm{mm}$ thick aluminium pipe and held in place by two cylindrical holders designed to host a circular PCB with a SiPM and its support circuitry. The holder has a central hole to allow for fiber-to-SiPM alignment and optical coupling by means of a tiny grease drop, and two side grooves to allow for the passage of cables. Two light-tight rubber caps complete the setup, with three cables coming out of one single side of detector for the common voltage bias and the two output signals. A sketch and three pictures of the SciFi detector components are shown in Fig. 7.

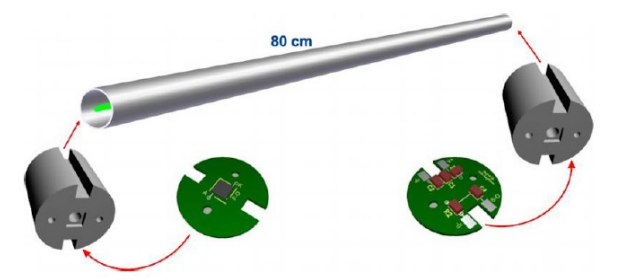

(a)
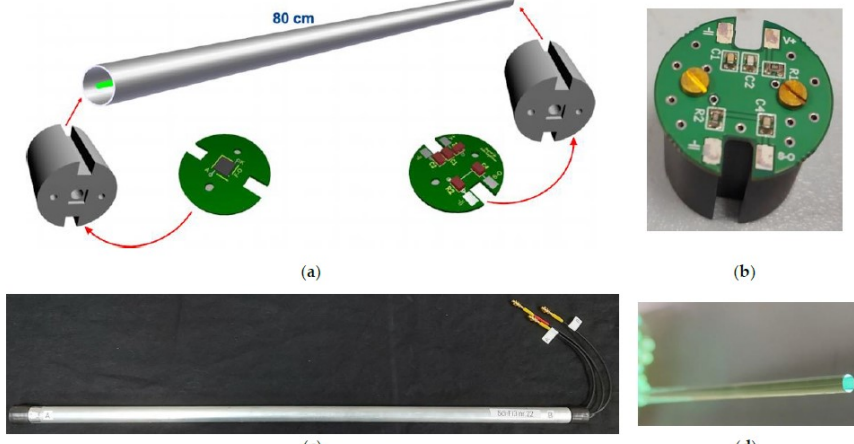

(b)

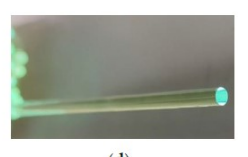

Fig. 7. (a) Sketch of the components of the SciFi detector assembly; (b) A fiber holder with the SiPM PCB in place; (c) A detector fully assembled; (d) A $3 \mathrm{~mm}$ diameter fiber.

The operating principle is the following: whenever a gammaray interacts with the fiber it deposits a variable amount of kinetic energy, mainly due to the Compton effect, which gives rise to a short flash of scintillation light. A fraction of the scintillation photons is trapped into the fiber and propagates toward both ends. If the number of these photons is large enough to produce a signal above a predefined threshold simultaneously on both fiber ends, such a coincidence event is considered as the detection of a gamma-ray and can be counted.

The chosen SiPM for these detectors, after careful evaluation and testing, is the MicroFC-30035-SMT, produced by ON Semiconductor [13]. For all the tests described was used of a homemade voltage amplifier $(200 \mathrm{x}$ gain and $4 \mathrm{GHz}$ bandwidth). The SiPM bias was set at a $2.5 \mathrm{~V}$ overvoltage, i.e., $27 \mathrm{~V}$ bias. A threshold at $175 \mathrm{mV}$ was used that corresponds to $\approx 3.5$ photons and reduces the noise rate by three orders of magnitude down to about 350 counts per second. As the signals have a duration of about $30 \mathrm{~ns}$, a duration of $100 \mathrm{~ns}$ for the coincidence window between the two SiPMs makes the probability of spurious coincidences negligible.

The chosen scintillating fiber, after GEANT4 simulation, evaluation and testing, is the $3 \mathrm{~mm}$ diameter $\mathrm{BCF}-20$ produced by Saint Gobain [14].

\section{A. Test results}

In order to characterize the detectors several tests were 
made with standard laboratory gamma sources and a high activity gamma and neutron source. The electronic setup was very simple: the outputs of the SiPM amplifiers were connected to two discriminators, with threshold set at $175 \mathrm{mV}$. The two logic outputs were used as inputs to a coincidence unit with a $100 \mathrm{~ns}$ window, and the final output was sent to a counter.

The validation of the detector behaviour was performed by means of a pointlike ${ }^{22} \mathrm{Na}$ source of activity $\mathrm{A}=42 \pm 2 \mathrm{kBq}$ which emits gamma-rays of $1274 \mathrm{keV}$. The source was placed at several distances from the fiber, in the range of few centimeters, and the number of detected gamma-rays was recorded. The observed count rate as function of the distance between the point-like ${ }^{22} \mathrm{Na}$ source and the $3 \mathrm{~mm}$ diameter fiber with $1 / \mathrm{r}$ fit is shown in Fig. 8.

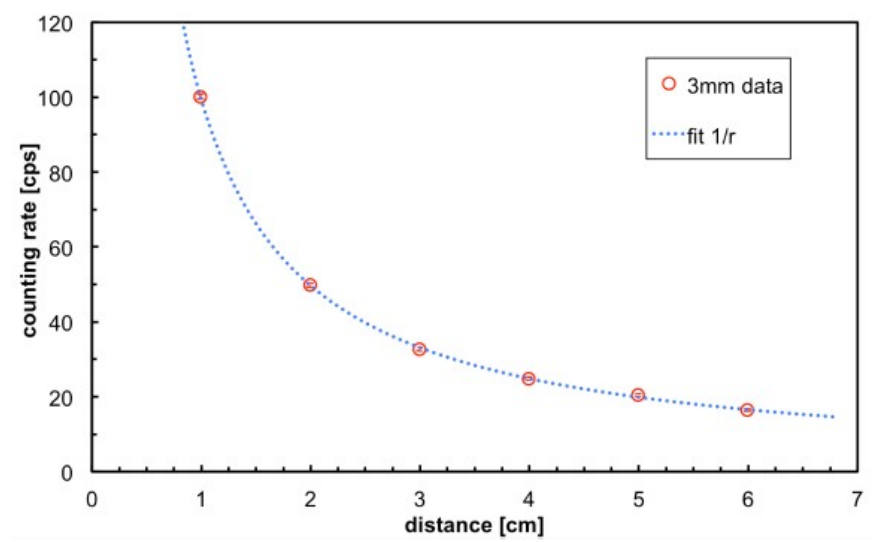

Fig. 8. Measured count rate vs distance between the point like $22 \mathrm{Na}$ source and the $3 \mathrm{~mm}$ diameter fiber.

To verify the uniformity of the response within the set of 36 SciFi detectors, ${ }^{137} \mathrm{Cs}$ gamma source was used, with activity $\mathrm{A}=1.49 \pm 0.07 \mathrm{Mbq}$, and a detector holder that kept the midpoint of the fiber at a distance $d=52 \mathrm{~cm}$ from the source. The counting rate of each detector was measured in $200 \mathrm{~s}$, as well as the background rate and the signal-to-background ratio, and the results are plotted in Fig. 9.

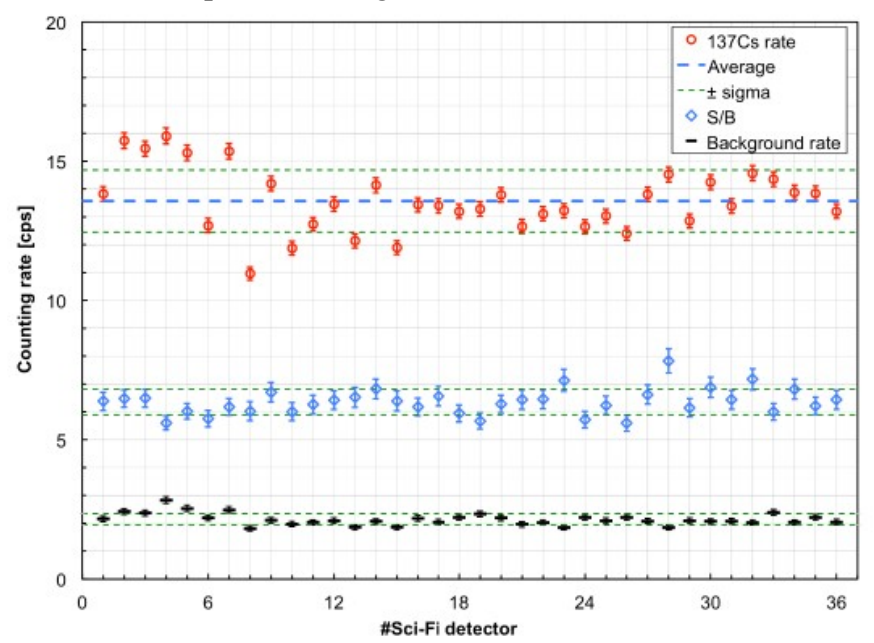

Fig. 9. The counting rate measured with the ${ }^{137} \mathrm{Cs}$ the background rate and the signal to background ratio of the $36 \mathrm{SciFi}$ detectors.

For a series of tests of the SciFi detectors, the same previously mentioned AmBe source was used. As it also emits a wide spectrum of gamma rays it represents a realistic setup recalling the emission from a drum of radioactive waste. All the SciFi detectors were tested on top of the source box, and the measured counting rate, the background and the signal-tobackground ratio are reported in Fig. 10.

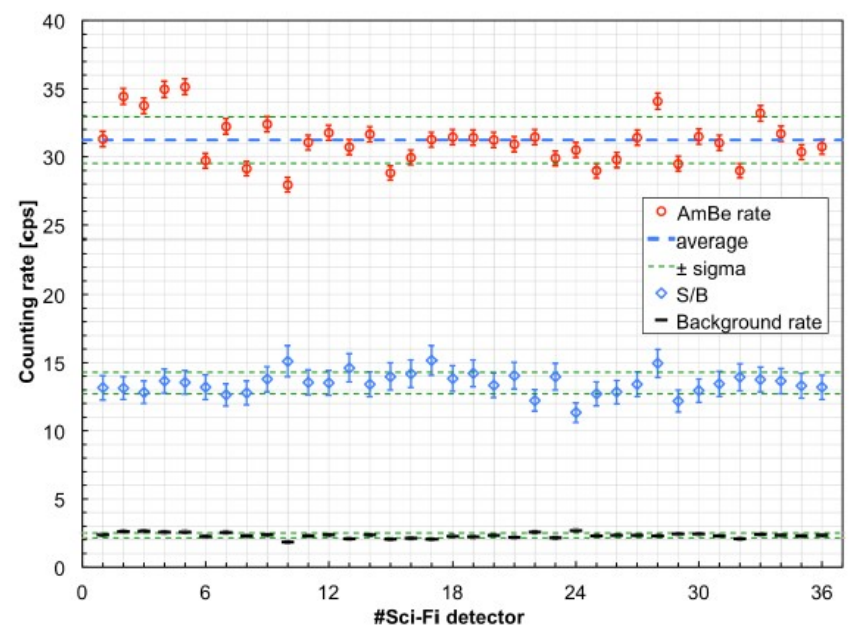

Fig. 10. The counting rate measured on top of the AmBe source box the background rate and the signal to background ratio of the $36 \mathrm{SciFi}$ detectors

For each detector, the ratio between the observed count rates with ${ }^{137} \mathrm{Cs}$ and the $\mathrm{AmBe}$ sources, respectively, was also calculated. The results, plotted in Fig. 11, indicate a quite reasonably constant behaviour of this ratio.

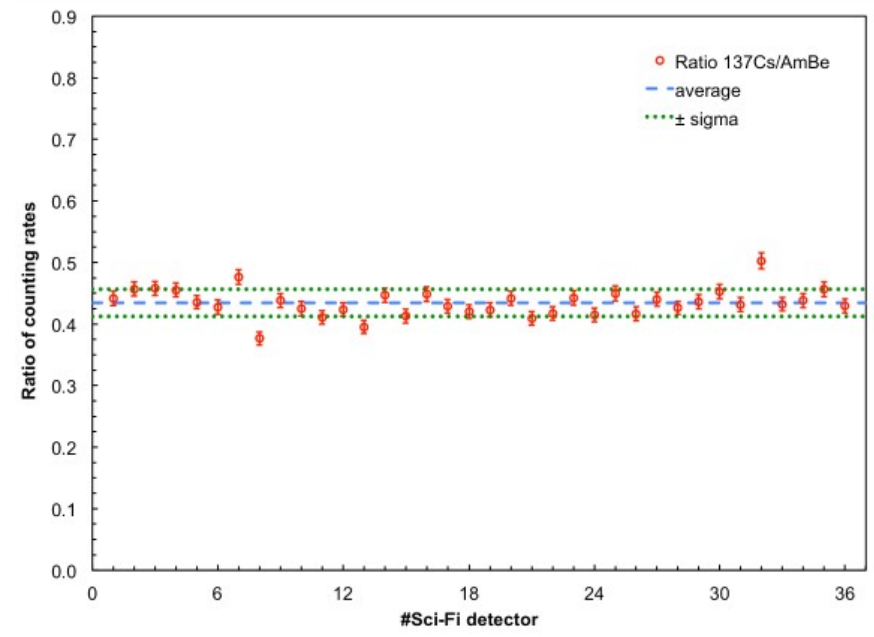

Fig. 11. Ratio between the observed counting rates respectively with the ${ }^{137} \mathrm{Cs}$ and the AmBe sources.

\section{DISCUSSION}

The dimension of a standard $220 \mathrm{~L}$ radioactive waste drum is $86 \mathrm{~cm}$ height and $57 \mathrm{~cm}$ diameter with a steel/polyethylene matrix (67/33\% in mass). A possible arrangement of the SiLiF and SciFi detectors around such a radwaste drums is shown in Fig. 12. In the MICADO perspective the drums will be first characterized with several methods based on active and passive neutron and gamma ray measurements, then the monitoring detectors will be used to check the longer-term stability of their radiological behavior. Possible counting asymmetries between the detectors would signal an asymmetry in the radioisotopes distribution or, should this 
occur afterwards, a change in the internal structure of the radwaste package. Obviously, for a more precise evaluation of the sensitivity one should take into account also the background from the neighboring drums.

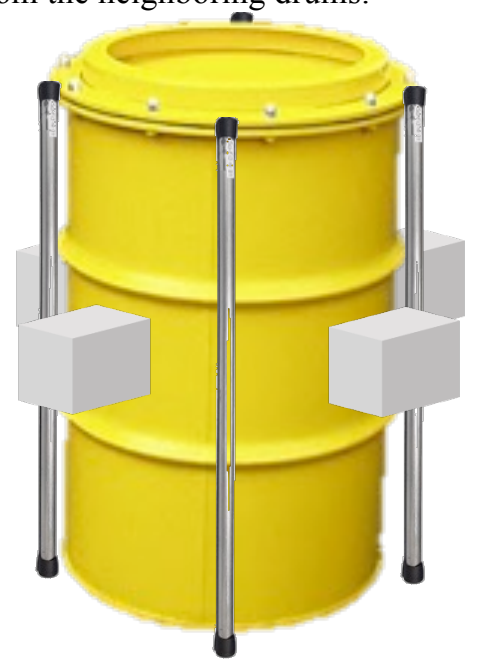

Fig. 12. Possible arrangement of the SiLiF and SciFi detectors on the radwaste drum.

The simulations allowed us to demonstrate the suitability of SiLiF and SciFi technology for the monitoring of radwaste drums that contain typical radioactive elements such as ${ }^{60} \mathrm{Co}$ $\mathrm{kBq},{ }^{133} \mathrm{Ba},{ }^{134} \mathrm{Cs},{ }^{137} \mathrm{Cs},{ }^{152} \mathrm{Eu},{ }^{154} \mathrm{Eu},{ }^{241} \mathrm{Am}$ and ${ }^{240} \mathrm{Pu}$.

The tests carried out have shown that SiLiF detectors can be fruitfully employed to detect neutrons, in a wide energy range and with excellent gamma rejection, and that the SciFi detectors can be successfully employed to detect and count gamma rays in a broad energy range. These results prove that SiLiF and SciFi detectors can successfully be employed in the MICADO project in order to demonstrate the reliability of neutron and gamma-ray monitoring in a medium-long-term storage of radioactive waste drums.

One last point concerns the possible radiation damage of silicon in a radiation field. The radiation hardness claimed by the manufacturer of the silicon is up to $10^{13} \mathrm{n} / \mathrm{cm}^{2}$. It is known that the main damage comes from fast neutrons which can dislocate silicon atoms thus creating defects in the semiconductor lattice. However, even considering the total neutron flux on the detectors, a life longer than hundreds of years is to be foreseen under these conditions. Indeed, as observed in reference [16], similar detectors were exposed to a fast neutron fluence of $3 \cdot 10^{10} / \mathrm{cm}^{2}$ without appreciable changes in their characteristics.

\section{CONCLusions}

The described tests, measurements and simulations have allowed us to prove the suitability of the SiLiF and SciFi technology for monitoring of radwaste drums.
The $32 \mathrm{SiLiF}$ and $36 \mathrm{SciFi}$ detectors we built have a reasonably uniform behavior, considering their robustness, low cost, and simple construction based on commercial components. We conclude that the SiLiF and SciFi detectors are promising candidates for low and intermediate level radioactive waste monitoring, and they are going to be tested soon in a real environment within the MICADO project.

\section{REFERENCES}

[1] MICADO Project. https://www.micado-project.eu/

[2] Italy in MICADO WP7. https://www.micado-project.eu/news/particles-ofitalianinnovation-project/

[3] Cosentino, L.; Ducasse, Q.; Giuffrida, M.; Lo Meo, S.; Longhitano, F.; Marchetta, C.; Massara, A.; Pappalardo, A.; Passaro, G.; Russo, S.; et al. SiLiF Neutron Counters to Monitor Nuclear Materials in the MICADO Project. Sensors 2021, 21, 2630. https://doi.org/10.3390/s21082630

[4] Cosentino, L.; Giuffrida, M.; Lo Meo, S.; Longhitano, F.; Pappalardo, A.; Passaro, G.; Finocchiaro, P. Gamma-Ray Counters to Monitor Radioactive Waste Packages in the MICADO Project. Instruments 2021, 5, 19. https://doi.org/10.3390/

[5] Finocchiaro, P. DMNR: A new concept for real-time online monitoring of short and medium term radioactive waste. In Radioactive Waste: Sources, Types and Management; Nova Science Publishers: New York, NY, USA, 2011; pp. 1-40.

[6] Finocchiaro, P. Radioactive Waste: A System for Online Monitoring and Data Availability. Nucl. Phys. News 2014, 24, 34.

[7] Cosentino, L.; Calì, C.; De Luca, G.; Guardo, G.; Litrico, P.; Pappalardo, A.; Piscopo, M.; Scirè, C.; Scirè, C.; Scirè, S.; et al. RealTime Online Monitoring of Radwaste Storage: A Proof-of-Principle Test Prototype. IEEE Trans. Nucl. Sci. 2012, 59, 1426-1431.

[8] Finocchiaro, P.; Ripani, M. RadioactiveWaste Monitoring: Opportunities from New Technologies. In Proceedings of the IAEA International Conference on Physical Protection of Nuclear Material and Nuclear Facilities, IAEA-CN-254/117, Vienna, Austria, 13-17 November 2017.

[9] Henzlova, D.; Kouzes, R.; McElroy, R.; Peerani, P.; Aspinall, M.; Baird, K.; Bakel, A.; Borella, M.; Bourne, M.; Bourva, L.; et al. Current status of $3 \mathrm{He}$ alternative technologies for nuclear safeguards. NNSA USDOE EURATOM, LA-UR-15-21201 2015 doi:10.2172/1227248.

[10] Barbagallo, M.; Cosentino, L.; Forcina, V.; Marchetta, C.; Pappalardo, A.; Peerani, P.; Scire, C.; Scire, S.; Schillaci, M.; Vaccaro, S.; et al. Thermal neutron detection using a silicon pad detector and $6 \mathrm{LiF}$ removable converters. Rev. Sci. Instrum. 2013, 84, 033503.

[11] Pappalardo, A.; Barbagallo, M.; Cosentino, L.; Marchetta, C.; Musumarra, A.; Scirè,C.; Scirè, S.; Vecchio, G.; Finocchiaro, P. Characterization of the silicon+6LiF thermal neutron detection technique. Nucl. Instrum. Methods Phys. Res. Sect. A 2016, 810, 6.

[12] Fasso, A. et al. P.R. FLUKA: A Multi-Particle Transport Code; CERN Technical Report No. INFN/TC_05/11; CERN-2005-10; 2005.

[13] ON Semiconductor https://www.onsemi.com/products/sensors/siliconphotomultipliers-sipm

[14] Saint Gobain Scintillating Fibers. Available online: https://www.crystals.saint-gobain.com/products/scintillating-fiber

[15] Nordlund, K.; Zinkle, S.J.; Sand, A.E.; Granberg, F.; Averback, R.S.; Stoller, R.E.; Suzudo, T.; Malerba, L.; Banhart, F.; Weber, W.J.; et al. Primary radiation damage: A review of current understanding and models. J. Nucl. Mater. 2018, 512, 450.

[16] Amaducci, S.; Cosentino, L.; Barbagallo, M.; Colonna, N.; Mengoni, A.; Massimi, C.; Meo, S.L.; Finocchiaro, P.; Aberle, O.; Andrzejewski, J.; et al. Measurement of the $235 \mathrm{U}(\mathrm{n}, \mathrm{f})$ cross section relative to the $6 \mathrm{Li}(\mathrm{n}, \mathrm{t})$ and $10 \mathrm{~B}(\mathrm{n}, \alpha)$ standards from thermal to $170 \mathrm{keV}$ neutron energy range at n TOF. Eur. Phys. J. A 2019, 55, 120. 